\title{
Can profiles of poly- and Perfluoroalkyl substances (PFASs) in human serum provide information on major exposure sources?
}

Xindi C. Hu ${ }^{1,2^{*}}$, Clifton Dassuncao ${ }^{1,2}$, Xianming Zhang ${ }^{2}$, Philippe Grandjean ${ }^{1,3}$, Pál Weihe ${ }^{4}$, Glenys M. Webster ${ }^{5}$, Flemming Nielsen ${ }^{3}$ and Elsie M. Sunderland ${ }^{1,2}$

\begin{abstract}
Background: Humans are exposed to poly- and perfluoroalkyl substances (PFASs) from diverse sources and this has been associated with negative health impacts. Advances in analytical methods have enabled routine detection of more than 15 PFASs in human sera, allowing better profiling of PFAS exposures. The composition of PFASs in human sera reflects the complexity of exposure sources but source identification can be confounded by differences in toxicokinetics affecting uptake, distribution, and elimination. Common PFASs, such as perfluorooctanoic acid (PFOA), perfluorooctane sulfonic acid (PFOS) and their precursors are ubiquitous in multiple exposure sources. However, their composition varies among sources, which may impact associated adverse health effects.

Methods: We use available PFAS concentrations from several demographic groups in a North Atlantic seafood consuming population (Faroe Islands) to explore whether chemical fingerprints in human sera provide insights into predominant exposure sources. We compare serum PFAS profiles from Faroese individuals to other North American populations to investigate commonalities in potential exposure sources. We compare individuals with similar demographic and physiological characteristics and samples from the same years to reduce confounding by toxicokinetic differences and changing environmental releases.

Results: Using principal components analysis (PCA) confirmed by hierarchical clustering, we assess variability in serum PFAS concentrations across three Faroese groups. The first principal component (PC)/cluster consists of C9-C12 perfluoroalkyl carboxylates (PFCAs) and is consistent with measured PFAS profiles in consumed seafood. The second $\mathrm{PC} /$ cluster includes perfluorohexanesulfonic acid (PFHXS) and the PFOS precursor $\mathrm{N}$-ethyl perfluorooctane sulfonamidoacetate (N-EtFOSAA), which are directly used or metabolized from fluorochemicals in consumer products such as carpet and food packaging. We find that the same compounds are associated with the same exposure sources in two North American populations, suggesting generalizability of results from the Faroese population.

Conclusions: We conclude that PFAS homologue profiles in serum provide valuable information on major exposure sources. It is essential to compare samples collected at similar time periods and to correct for demographic groups that are highly affected by differences in physiological processes (e.g., pregnancy). Information on PFAS homologue profiles is crucial for attributing adverse health effects to the proper mixtures or individual PFASs.
\end{abstract}

Keywords: Serum, Fish and shellfish, Consumer products, Source attribution, Homologues, Perfluoroalkyl carboxylates (PFCAs)

\footnotetext{
* Correspondence: xhu@mail.harvard.edu

${ }^{1}$ Harvard T.H. Chan School of Public Health, Boston, MA 02215, USA

${ }^{2}$ Harvard John A. Paulson School of Engineering and Applied Sciences,

Harvard University, 128 Pierce Hall, Cambridge, MA 02138, USA

Full list of author information is available at the end of the article
} 


\section{Background}

Poly- and perfluoroalkyl substances (PFASs) are widely used consumer and industrial chemicals that are now detectable in virtually all human populations [1-6]. Exposure to PFASs has been associated with many adverse health effects including developmental, metabolic and immune disorders, and increased risk of certain cancers [7-9]. PFAS exposure sources are diverse and include dust, food, drinking water, and many consumer products such as food packaging, outdoor gear, dental floss, carpets and furniture coatings [10-12]. Human populations are therefore exposed to different PFAS mixtures. The relative importance of different PFAS exposure sources has proven difficult to discern, both within and across populations. This information is essential for attributing adverse effects to particular PFASs or mixtures and for prioritizing actions to minimize health risks. Complex mixtures of PFASs in human sera may be further altered by variability in toxicokinetics. Improved classification of particular PFAS mixtures may allow chemical fingerprinting of PFASs in human sera and eventually lead to characterization of major causative profiles within populations and across demographic groups.

The relative importance of different PFAS exposure sources can be highly variable across populations and locations. Exposures from seafood consumption are important in many coastal communities [4, 13-15]. Other food items of animal origin have been identified as important contributors to exposure in a few other populations [16, 17]. Children and toddlers are more highly exposed to certain PFASs due to trans-placental transfer, breastfeeding, and frequent hand-to-mouth contact leading to ingestion of house dust [18-20]. Other populations may be exposed to specific PFASs from drinking water $[21,22]$. Exposures estimated from measured concentrations in food, dust, and drinking water can be imprecise due to large inter-individual differences in behavior and wide ranges in environmental concentrations [23]. This imprecision is compounded when environmental measurements are not available for a specific study population and must be inferred from other studies. For example, Lorber and Egeghy [23] modeled US population exposures using PFAS concentrations in foods from another population [24] and resulting dietary perfluorooctanoic acid (PFOA) intake estimates ranged by more than one order of magnitude [23]. Others have developed multicompartment pharmacokinetic models for individual PFASs that reproduce observed serum concentrations [25, 26]. However, such modeling approaches are typically data-intensive and focus on individual PFASs rather than chemical mixtures. Toxicokinetic data needed to parameterize such models are limited for PFASs other than PFOS, PFOA and PFHxS.
Serum concentrations of PFASs reflect accumulated exposures to multiple compounds that occurred simultaneously, providing a composite measure of uptake from all pathways and sources [23, 27]. Previous work has successfully used congener composition within chemical classes such as polychlorinated biphenyls (PCBs) to identify the origin of major human exposure sources [28-30]. Applying such techniques to PFASs is complicated by dramatic shifts in production over time and the complex metabolism of PFAS precursors. This means that differences in exposure can be inferred only from samples collected at similar time periods, and physiological differences must be carefully considered. Previously, we showed that the measured PFAS composition in surface water provides useful information on sources of environmental pollution [31]. Here, we discuss the potential feasibility of extending this approach to consider human sera and the caveats associated with such methods.

The main objective of this study is to assess exposure information that can be derived from serum-PFAS composition. We analyzed a suite of 19 PFASs in archived serum samples from past epidemiological studies in the Faroe Islands, a North Atlantic fishing community with a wide-range of PFAS exposures [14, 32]. We compare the measured composition of detectable PFASs in serum from Faroese women, children and men using multivariate statistical tools to identify clustering of major PFASs. We consider potential confounding due to inter-individual differences in toxicokinetics and elimination pathways, and test generalizability of information derived from the Faroese cohort using serum PFAS data from two other North American populations [33, 34].

\section{Methods}

\section{Sample selection}

We selected archived serum samples from adult men, women and children in the Faroe Islands that were available for overlapping time periods. The Faroese are a fairly homogeneous population consisting of individuals of mainly Scandinavian descent. Sera from the same children $(n=52)$ were collected at age 7 between 2004-05 and at age 13 between 2011-12 as part of a larger prospective birth cohort described elsewhere [32]. Adult male serum samples $(n=10)$ were collected in 2006 from individuals who reported participating in pilot whale harvesting (whalers) and were thus expected to have the highest PFAS exposures from seafood [14]. Women $(n=52)$ were part of a different prospective cohort established in 2007. Blood samples were collected 2 weeks after singleton delivery in 2007-08 [35, 36]. For children, the 2004-05 serum collection was compared with Faroese whalers and women from the overlapping time periods, while the 2011-12 serum collection was compared to North American children of the same age from the same time period, as further described below. 
Dietary questionnaire data on seafood consumption, such as the number of whale meat meals per month, were available for all Faroese individuals. Imprecision in dietary survey data is well established due to several types of recall bias [37]. Measured hair mercury concentrations provide an alternative empirical proxy for seafood exposure because methylmercury is almost exclusively from fish, shellfish and marine mammal consumption [37]. Thus, we use previously reported hair mercury data from Faroese subjects as an additional indicator for seafood consumption [38]. All mothers and other participants provided written informed consent, and study protocols were reviewed and approved by the Faroese ethical review committee and the Institutional Review Board of Harvard T.H. Chan School of Public Health.

\section{Sample analysis for PFASs}

Blood samples $(10 \mathrm{~mL})$ from Faroese individuals were allowed to clot for approximately $30 \mathrm{~min}$ and spun at $2000 \mathrm{~g}$ for $10 \mathrm{~min}$ to separate the serum. Serum samples were transferred into polypropylene cryogenic storage vials $\left(2.5\right.$ or $5 \mathrm{~mL}$ ) and stored at $-80{ }^{\circ} \mathrm{C}$ until analysis. PFASs (Table 1) were analyzed by online solid-phase extraction and high-pressure liquid chromatography with tandem mass spectrometry (HPLC-MS/MS) [39]. For each sample, $150 \mu \mathrm{L}$ serum was pipetted into a $2 \mathrm{~mL}$ centrifuge tube. Internal standard solution $\left(30 \mu \mathrm{L}, 50 \mathrm{ng} \mathrm{mL}^{-1}\right.$ for perfluorooctane sulfonic acid (PFOS) and $20 \mathrm{ng} \mathrm{mL}^{-1}$ for other PFASs, Wellington Laboratories, Canada), and methanol $(120 \mu \mathrm{L})$ were added prior to mixing on a whirl mixer. Calibration solutions were prepared in serum from newborn calves (Biological Industries, Israel) because it matches the matrix of human serum and contains low levels of PFASs. Solutions spanned a concentration range from 0.050 to $100 \mathrm{ng}$ PFASs $\mathrm{mL}^{-1}$ serum for all analytes. Both calibration solutions and samples were whirl mixed and centrifuged at $15,000 \mathrm{rpm}$ for $20 \mathrm{~min}$. $160 \mu \mathrm{L}$ of the supernatant was transferred to a polypropylene autosampler vial. Formic acid $(400 \mu \mathrm{L}, 0.1 \mathrm{M})$ was added and the solution was mixed on a whirl mixer and placed in the autosampler for injection into HPLC-MS/MS system. A volume of $400 \mu \mathrm{L}$ was injected into the on-line solid-phase extraction column.

In each analytical series ( 50 samples), quality control serum samples, calibration standards, and reagent and serum blanks were included to ensure the accuracy and reliability of data. Within-batch and between-batch coefficient of variations were lower than $8.9 \%$ and $12.9 \%$ for

Table 1 List of quantified PFASs in Faroese serum samples and their limits of detection

\begin{tabular}{|c|c|c|c|c|}
\hline Analyte & Acronym & $\begin{array}{l}\text { Carbon-chain } \\
\text { length }\end{array}$ & Molecular ion & $\begin{array}{l}\mathrm{LOD} \\
(\mathrm{ng} / \mathrm{mL})\end{array}$ \\
\hline \multicolumn{5}{|l|}{ Carboxylic acids (PFCAs) } \\
\hline Perfluorobutanoic acid & PFBA & $C-4$ & $\mathrm{~F}\left(\mathrm{CF}_{2}\right)_{3} \mathrm{CO}_{2}^{-}$ & 0.1 \\
\hline Perfluoropentanoic acid & PFPeA & $C-5$ & $\mathrm{~F}\left(\mathrm{CF}_{2}\right)_{4} \mathrm{CO}_{2}^{-}$ & 0.05 \\
\hline Perfluorohexanoic acid & PFHXA & $C-6$ & $\mathrm{~F}\left(\mathrm{CF}_{2}\right)_{5} \mathrm{CO}_{2}^{-}$ & 0.05 \\
\hline Perfluoroheptanoic acid & PFHpA & $C-7$ & $\mathrm{~F}\left(\mathrm{CF}_{2}\right)_{6} \mathrm{CO}_{2}^{-}$ & 0.03 \\
\hline Perfluorooctanoic acid & PFOA & $C-8$ & $\mathrm{~F}\left(\mathrm{CF}_{2}\right)_{7} \mathrm{CO}_{2}^{-}$ & 0.03 \\
\hline Perfluorononanoic acid & PFNA & C-9 & $\mathrm{F}\left(\mathrm{CF}_{2}\right)_{8} \mathrm{CO}_{2}^{-}$ & 0.03 \\
\hline Perfluorodecanoic acid & PFDA & $C-10$ & $\mathrm{~F}\left(\mathrm{CF}_{2}\right)_{9} \mathrm{CO}_{2}^{-}$ & 0.03 \\
\hline Perfluoroundecanoic acid & PFUnDA & $C-11$ & $\mathrm{~F}\left(\mathrm{CF}_{2}\right){ }_{10} \mathrm{CO}_{2}^{-}$ & 0.03 \\
\hline Perfluorododecanoic acid & PFDoDA & $C-12$ & $\mathrm{~F}\left(\mathrm{CF}_{2}\right)_{11} \mathrm{CO}_{2}^{-}$ & 0.05 \\
\hline \multicolumn{5}{|l|}{ Sulfonic acids (PFSAs) } \\
\hline Perfluorobutane sulfonic acid & PFBS & $C-4$ & $\mathrm{~F}\left(\mathrm{CF}_{2}\right)_{4} \mathrm{SO}_{3}^{-}$ & 0.1 \\
\hline Perfluorohexane sulfonic acid & PFHXS & $C-6$ & $\mathrm{~F}\left(\mathrm{CF}_{2}\right)_{6} \mathrm{SO}_{3}^{-}$ & 0.05 \\
\hline Perfluoroheptane sulfonic acid & PFHpS & $C-7$ & $\mathrm{~F}\left(\mathrm{CF}_{2}\right)_{7} \mathrm{SO}_{3}^{-}$ & 0.03 \\
\hline Linear perfluorooctane sulfonic acid & nPFOS & $C-8$ & $\mathrm{~F}\left(\mathrm{CF}_{2}\right)_{8} \mathrm{SO}_{3}^{-}$ & 0.03 \\
\hline Branched perfluorooctane sulfonic acid & brPFOS & $C-8$ & $\mathrm{~F}\left(\mathrm{CF}_{2}\right)_{8} \mathrm{SO}_{3}^{-}$ & 0.03 \\
\hline Perfluorodecane sulfonic acid & PFDS & $C-10$ & $\mathrm{~F}\left(\mathrm{CF}_{2}\right)_{10} \mathrm{SO}_{3}^{-}$ & 0.03 \\
\hline \multicolumn{5}{|l|}{ PFOS Precursors (PreFOS) } \\
\hline N-ethyl perfluorooctane sulfonamidoacetate & N-EtFOSAA & $C-8$ & $\mathrm{~F}\left(\mathrm{CF}_{2}\right)_{8} \mathrm{SO}_{2} \mathrm{~N}\left(\mathrm{C}_{2} \mathrm{H}_{5}\right) \mathrm{CH}_{2} \mathrm{CO}_{2}^{-}$ & 0.03 \\
\hline $\mathrm{N}$-methyl perfluorooctane sulfonamidoacetate & N-MeFOSAA & $C-8$ & $\mathrm{~F}\left(\mathrm{CF}_{2}\right)_{8} \mathrm{SO}_{2} \mathrm{~N}\left(\mathrm{CH}_{3}\right) \mathrm{CH}_{2} \mathrm{CO}_{2}^{-}$ & 0.03 \\
\hline Linear perfluorooctane sulfonamide & nFOSA & $C-8$ & $\mathrm{~F}\left(\mathrm{CF}_{2}\right)_{8} \mathrm{SO}_{2} \mathrm{NH}_{2}$ & 0.03 \\
\hline Branched perfluorooctane sulfonamide & brFOSA & $C-8$ & $\mathrm{~F}\left(\mathrm{CF}_{2}\right)_{8} \mathrm{SO}_{2} \mathrm{NH}_{2}$ & 0.03 \\
\hline
\end{tabular}


all analytes. All serum samples were analyzed for 19 PFASs and the limit of detection (LOD) for PFASs was 0.03-0.1 ng $\mathrm{mL}^{-1}$ (Table 1). Four analytes (PFBA, PFPeA, PFHxA and PFBS) were below the LOD for all Faroese children and women, and were therefore excluded as target analytes for the Faroese whaling men.

\section{Other study populations}

We compared serum PFAS data from the Faroese to exposure information from two North American cohorts studied at overlapping time periods. Most consumer products that are potential sources of PFAS exposure in the Faroe Islands are imported. Thus, use of consumer products in the Faroes is expected to result in similar exposures to PFASs as in European and North American populations. PFASs with longer carbon chain length are well established to have greater propensity to bioaccumulate in freshwater and marine fish, shellfish and marine mammals [40, 41], providing a characteristic exposure signature for seafood consumers across many populations [42-44].

Our analysis used serum PFAS data from the US National Health and Nutrition Examination Survey (NHANES), a cross-sectional survey of the U.S general population [34]. Questionnaire data and serum PFAS levels for NHANES are available for $n=2120$ individuals in 2005-06 (age $>12$ years, 50.6\% female) and for $n=$ 1751 individuals in 2011-12 (age > 12 years, $48.7 \%$ female). We downloaded serum PFAS and seafood consumption data, along with other relevant predictors, such as data on demographic characteristics and reproductive health, and merged them into one file [34]. Laboratory methods are available on the Center for Disease Control and Prevention $(\mathrm{CDC})$ website and the LOD for different PFASs ranged between $0.1-0.4 \mathrm{ng} \mathrm{mL}^{-1}$ [45].

We also synthesized exposure information and behavior data from the Chemicals, Health and Pregnancy (CHirP) cohort established in Vancouver, Canada [46, 47]. Between December 2006 and June 2008, the CHirP study enrolled and collected blood samples from 152 pregnant women in their first trimester. Data on potential covariates such as demographic attributes, dietary habits, and use of consumer products were collected using online and in-person questionnaires between weeks 19 to 24 of the pregnancy. Serum samples were analyzed for 23 PFASs at ALS Laboratory in Edmonton, Canada by HPLC-MS/MS following a solid-phase extraction method similar to Kuklenyik et al. [46, 48]. The LOD in CHirP was higher than the Faroese and NHANES studies $\left(0.5 \mathrm{ng} \mathrm{mL}{ }^{-1}\right.$ for all PFASs in serum) and only four PFASs (PFOS, PFOA, PFNA and PFHxS) were detectable in more than $50 \%$ of participants. Participant characteristics of NHANES 2005-06 and CHirP are outlined in Additional file 1: Table S1 and Table S2.

\section{Statistical methods}

Multiple statistical techniques were used to handle the left censored data on serum PFAS concentrations. For the main analysis, we used simple substitution by the LOD multiplied by $1 / \sqrt{ } 2$. We then tested the impacts of this assumption using the robust regression on order statistics (ROS) method to impute the missing data containing multiple detection limits $[49,50]$. All 15 PFASs were frequently detected among Faroese whaling men $(60 \%$ $100 \%)$. We included six compounds with low frequency of detection in Faroese children $(12 \%-100 \%)$ and women (2\% - 27\%) because variability in their detection provides useful information on potential exposure sources.

For each PFAS, we used analysis of variance (ANOVA) to compare mean serum concentrations among Faroese whaling men, children, and women. Significant differences were identified using Tukey's honestly significant difference (HSD) tests with $\alpha=0.05$ ( $\mathrm{R}$ package agricolae) [51]. For Faroese children, we calculated Spearman correlation coefficients between serum PFAS concentrations and factors reflecting seafood consumption (hair mercury and whale consumption frequency).

We used principal component analysis (PCA) and hierarchical clustering to identify variability in PFAS profiles across Faroese men, women and children. PCA was applied to the covariance matrix of the PFAS concentration profiles ( $\mathrm{R}$ package FactoMineR) [52], and the number of principal components was based on those with an eigenvalue greater than one. Hierarchical clustering with Ward's method was used to investigate similarities among the serum PFAS profiles, defined as the squared Euclidean distance (R package stats) [53].

We matched the Faroese whaling men and women with individuals of similar demographic characteristics (age, gender, race) and physiological characteristics (postpartum) from NHANES 2005-06 who provided serum samples at approximately the same time period as the Faroese (Table 2). Selection of the same collection period, and similar demographic and physiological characteristics in these cohorts reduces confounding by changing production and environmental releases and toxicokinetic differences across demographic groups. A comparison with the data from Faroese children at age 7 was not possible because NHANES participants are 12 years and older. We thus used data from the same Faroese cohort in 2011-12 when they reached age 13 to compare to age matched US individuals from NHANES 2011-12. Related work suggests gender differences among young teens does not influence PFAS body burdens [54].

We selected 56 white men and 23 white women within one year-postpartum period from NHANES 2005-06 and 30 white children from NHANES 2011-12. We projected these NHANES population onto the principal 
Table $\mathbf{2}$ Characteristics of Faroese and NHANES individuals included in the PCA analysis

\begin{tabular}{|c|c|c|c|c|c|c|}
\hline & Men & & Children & & Women $^{*}$ & \\
\hline & Faroese & NHANES & Faroese & NHANES & Faroese & NHANES \\
\hline$n$ & 10 & 56 & 51 & 30 & 51 & 23 \\
\hline Sampling year & 2006 & 2005-2006 & $2011-2012$ & 2011-2012 & $2007-2008$ & $2005-2006$ \\
\hline Age & $47-80$ & $47-80$ & 13 & $12-14$ & $19-44$ & $19-44$ \\
\hline Race/ethnicity & White & White & White & White & White & White \\
\hline
\end{tabular}

"Note: Faroese women were two weeks postpartum and NHANES women were within one year postpartum

components derived from PCA analysis on the Faroese, and assign scores based on the original loadings. Unlike the Faroese serum PFASs measurements, NHANES data does not distinguish between linear and branched PFOS, and linear and branched FOSA. To compare to Faroese measurements, we thus assumed NHANES data contained $69 \%$ linear and 31\% branched based on literature values for Norwegian adults [55].

We developed multivariable regression models for the NHANES 2005-06 $(n=2120)$ and CHirP cohorts $(n=$ 151) based on measured PFAS levels in serum and different potential drivers of PFAS exposures such as seafood consumption, consumer product use and drinking water sources. We focused on compounds identified in the PCA unless the detection frequency was $<50 \%$. The multivariable regression model was used to predict serum levels of individual PFASs and thus differs from the PCA that selected for co-varying compounds. In NHANES, seafood consumption was derived from a 30day food frequency questionnaire.

Information on consumer product use is limited in NHANES. A study of household dust in NHANES 2005-06 includes data on floor cover, allowing us to infer the use of carpet cleaning products. Other variables include age, gender, race, household income, health factors such as menstrual status, and potential exposure sources such as tap water sources, past military service and country of birth [56].

The CHirP study collected detailed information on seafood consumption, and consumer product use including food packaging (popcorn bags, takeout and paper cups), carpet cleaner, car polishes, non-stick cookware, waterproof sprays and waxes. Frequencies were recorded in either categorical format such as "have used carpet repellent or not in the past 3 years," or continuous format such as "number of takeout meals per year in the year before pregnancy." We selected the best multivariable model of PFAS serum concentrations in the CHirP cohort using a supervised forward stepwise procedure.

We first tested linear and nonlinear effects of each independent variable on the log-transformed serum PFAS levels using the generalized additive models (GAMs, R package gam) with a cubic spline smoothing function [57].
Predictors with $p$-values below 0.05 were retained for inclusion in the subsequent multivariable models. We present results in tabular format for parametric terms, and in graphic format for smoothed terms. For regression results, we calculate percent difference in PFAS concentrations associated with each predictor by exponentiating regression coefficients, minus one, and multiplying by $100 \%$.

\section{Results}

\section{Serum PFAS profiles among Faroese individuals}

Serum PFAS profiles for Faroese individuals shown in Fig. 1 reflect combined exposures from different sources and toxicokinetic processes affecting elimination. The sum of PFASs measured here are statistically greater in whaling men, followed by children, and lowest in women (Fig. 1, one-way ANOVA, $p<0.001$ ). Sensitivity analyses using the ROS method to impute censored data generated similar results (Fig. S1). PFAS profiles of whaling men are dominated by PFOS, which accounts for almost $80 \%$ of the sum of PFASs (Fig. S2). Faroese children at age 7 have significantly elevated concentrations of PFHxS and N-EtFOSAA, compared to whaling men and women (Tukey HSD test, $\alpha=0.05$, Table 3, Fig. 1 ).

The first three components of the PCA explain $77 \%$ of the total variability in serum PFAS concentrations (Fig. 2a and Additional file 1 Figure S3; detailed loadings are provided in Additional file 1: Table S3). The three Faroese demographic groups have similar principal component scores within groups. Repeating the PCA analyses using the ROS method to impute censored data generated similar results (Additional file 1: Figure S4).

The first PCA component explains 51\% of the variability, and clearly separates whaling men from children and women. PFOS and C9-C12 PFCAs have high loadings on the first component and are distinct from the other PFASs. The first component includes compounds with both low and high detection frequency indicating this grouping is unlikely to be driven by detection rates.

The second component explains $17 \%$ of the variability in serum PFAS profiles and has high scores among Faroese children. PFHxS, PFHpA and two PFOS precursors, N-MeFOSAA and N-EtFOSAA have high loadings in this component of the PCA. The third component explains $9 \%$ of the variability. Two PFOS precursors, 


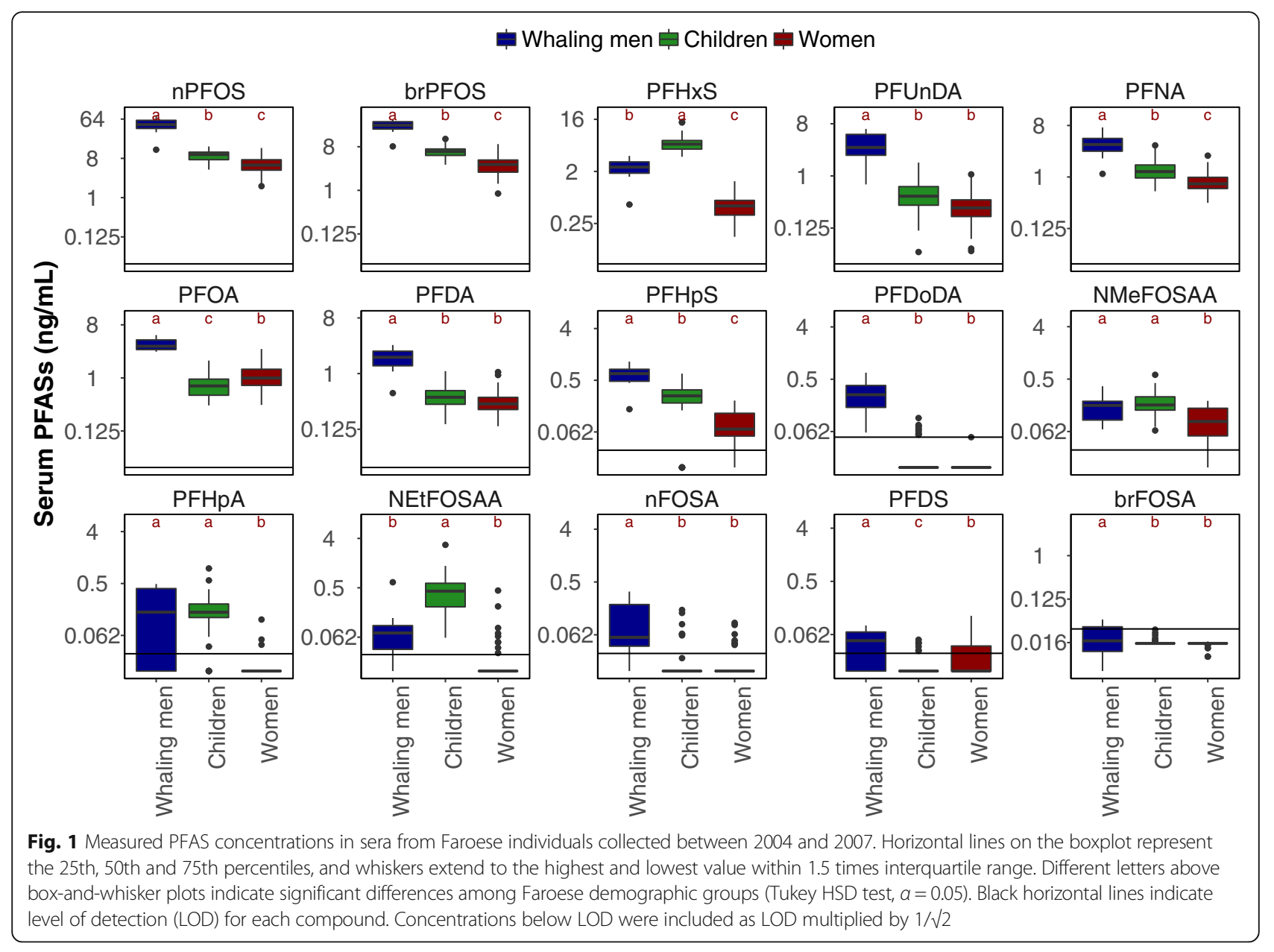

Table 3 PFAS concentrations ( $\left.n g \mathrm{~mL}^{-1}\right)$ measured in serum samples collected from Faroese whaling men $(M, n=10)$, children (C, $\left.n=51\right)$, and women $(W, n=51)$ between 2004-07

\begin{tabular}{|c|c|c|c|c|c|c|c|c|c|}
\hline \multirow[t]{2}{*}{ Analyte } & \multicolumn{3}{|c|}{ Detection Frequency (\%) } & \multicolumn{3}{|c|}{ Median } & \multicolumn{3}{|l|}{ Range } \\
\hline & $\mathrm{M}$ & C & W & $\mathrm{M}$ & C & W & $\mathrm{M}$ & C & W \\
\hline nPFOS & 100 & 100 & 100 & 47.8 & 9.82 & 5.59 & $12.66-76.04$ & $4.40-15.0$ & $1.84-13.8$ \\
\hline brPFOS & 100 & 100 & 100 & 22.4 & 6.38 & 3.43 & $8.13-28.83$ & $3.39-11.6$ & $0.86-9.08$ \\
\hline PFHXS & 100 & 100 & 100 & 2.38 & 5.94 & 0.5 & $0.53-3.71$ & $3.59-14.3$ & $0.15-1.35$ \\
\hline PFUnDA & 100 & 100 & 100 & 3.13 & 0.45 & 0.28 & $0.71-6.51$ & $0.05-1.7$ & $0.05-1.06$ \\
\hline PFNA & 100 & 100 & 100 & 3.76 & 1.24 & 0.76 & $1.14-7.39$ & $0.56-3.58$ & $0.35-2.37$ \\
\hline PFOA & 100 & 100 & 100 & 3.46 & 0.73 & 1.0 & $2.79-5.36$ & $0.34-1.97$ & $0.35-3.1$ \\
\hline PFDA & 100 & 100 & 100 & 1.85 & 0.41 & 0.32 & $0.48-2.91$ & $0.15-1.1$ & $0.14-1.06$ \\
\hline PFHpS & 100 & 90 & 86 & 0.65 & 0.28 & 0.09 & $0.16-1.06$ & $<\mathrm{LOD}-0.65$ & $<\mathrm{LOD}-0.22$ \\
\hline PFDoDA & 100 & 20 & 2 & 0.27 & 0.07 & 0.05 & $0.06-0.65$ & $<\mathrm{LOD}-0.11$ & $<\mathrm{LOD}-0.05$ \\
\hline N-MeFOSAA & 100 & 100 & 92 & 0.18 & 0.18 & 0.1 & $0.07-0.38$ & $0.06-0.59$ & $<\mathrm{LOD}-0.21$ \\
\hline PFHpA & 60 & 90 & 6 & 0.39 & 0.18 & 0.05 & $<\mathrm{LOD}-0.49$ & $<\mathrm{LOD}-0.92$ & $<\mathrm{LOD}-0.12$ \\
\hline $\mathrm{N}$-EtFOSAA & 90 & 100 & 18 & 0.08 & 0.43 & 0.07 & $<\mathrm{LOD}-0.63$ & $0.06-3.05$ & $<\mathrm{LOD}-0.45$ \\
\hline nFOSA & 80 & 14 & 14 & 0.08 & 0.07 & 0.05 & $<\mathrm{LOD}-0.34$ & $<\mathrm{LOD}-0.17$ & $<\mathrm{LOD}-0.1$ \\
\hline PFDS & 60 & 12 & 27 & 0.07 & 0.04 & 0.06 & $<\mathrm{LOD}-0.09$ & $<\mathrm{LOD}-0.05$ & $<\mathrm{LOD}-0.13$ \\
\hline brFOSA & 90 & 14 & 12 & 0.02 & 0.02 & 0.01 & $<\mathrm{LOD}-0.05$ & $<\mathrm{LOD}-0.03$ & $<\mathrm{LOD}-0.01$ \\
\hline 15 PFASS & & & & 87.8 & 26.2 & 12.9 & $26.9-129.1$ & $16.3-39.4$ & $5.01-25.9$ \\
\hline
\end{tabular}




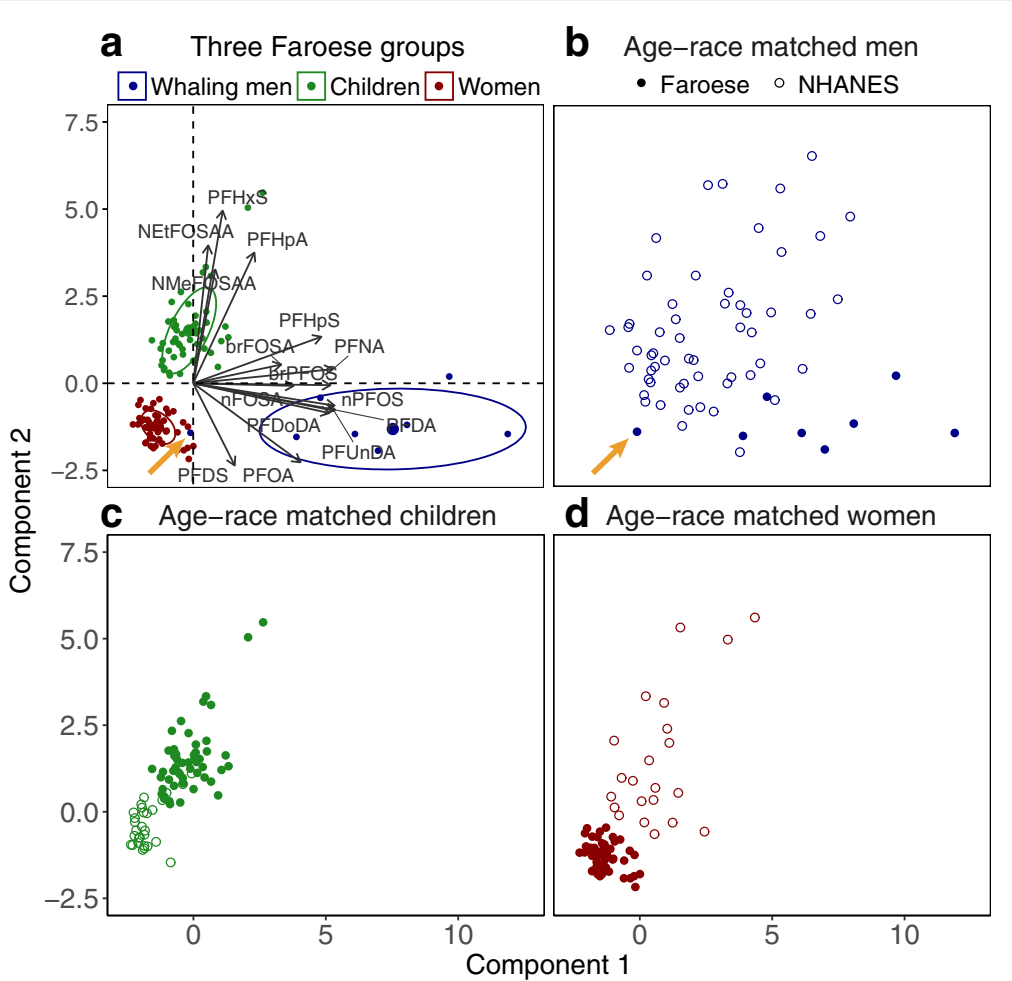

Fig. 2 Principal component analysis (PCA) of serum PFAS profiles for (a) Faroese whaling men, children and women, (b) Faroese whaling men and US men, (c) Faroese children and US children, (d) Faroese women and US women. In Panel (a) and (b), the orange arrow points to a whaling man who did not consume pilot whale meat in the year preceding serum collection. In Panel (b) - (d), the coordinates of each data point are computed as the raw data on serum PFASs weighted by the factor loadings derived from Panel (a)

nFOSA and brFOSA have high loadings in this component, but the three Faroese groups have similar scores on this component.

We compared PCA results for Faroese groups to their US counterparts with similar demographic and physiological characteristics with serum samples collected at the same time period to reduce temporal variability in the exposure signal and toxicokinetic differences (Fig. 2b-d). Faroese whaling men have higher scores on Component 1 when compared to white men from NHANES 2005-06 (Fig. 2b). Faroese children at age 13 (2011-12) have higher scores on Component 1 and Component 2 compared to age 13 white children in NHANES for the same year (Fig. $2 \mathrm{c})$. Figure $2 \mathrm{~d}$ shows the PCA results for mothers from the Faroe Islands and US white women, who are 19 to 44 years old and within the one year-postpartum period, from NHANES 2005-06. The mothers from the Faroes have lower scores on Component 1 and Component 2 compared to women in NHANES.

Similar to the PCA, results of hierarchical clustering clearly separate whaling men, children and women into distinct groups (Fig. 3). One whaling man who reported not eating any whale meat in the year prior to blood sample collection fell into the same cluster as women. Clustering of different PFASs is mostly consistent with
PCA results. The cluster associated with Faroese children (cluster 2) does not include N-MeFOSAA, which is instead associated with women (cluster 3). The composition of PFASs clustered with whaling men (cluster 1) does not include PFOA and PFDS, which are again associated with women. These differences likely occur because hierarchical clustering considers all available data, whereas the first three principal components of PCA only explain $77 \%$ of the variability in PFAS composition.

Figure 4 shows correlations between concentrations of PFASs measured in Faroese children and number of whale meat meals per month. Hair mercury concentrations, a proxy for magnitudes of seafood consumption, are significantly correlated with PFHpS, PFDA, PFOS and PFUnDA in the Faroese children (Spearman rho value: 0.35-0.61, Fig. 4). Correlations between reported whale meat consumption frequency and long-chain PFASs (such as PFDA, PFOS, and PFUnDA) are similar but slightly weaker than those observed for hair mercury and the same PFASs. This likely reflects recall bias associated with dietary survey data rather than a true difference [37].

\section{Predictors of PFASs in two north American populations} We found significant associations between shellfish consumption and serum levels of PFOS, PFOA, PFNA and 


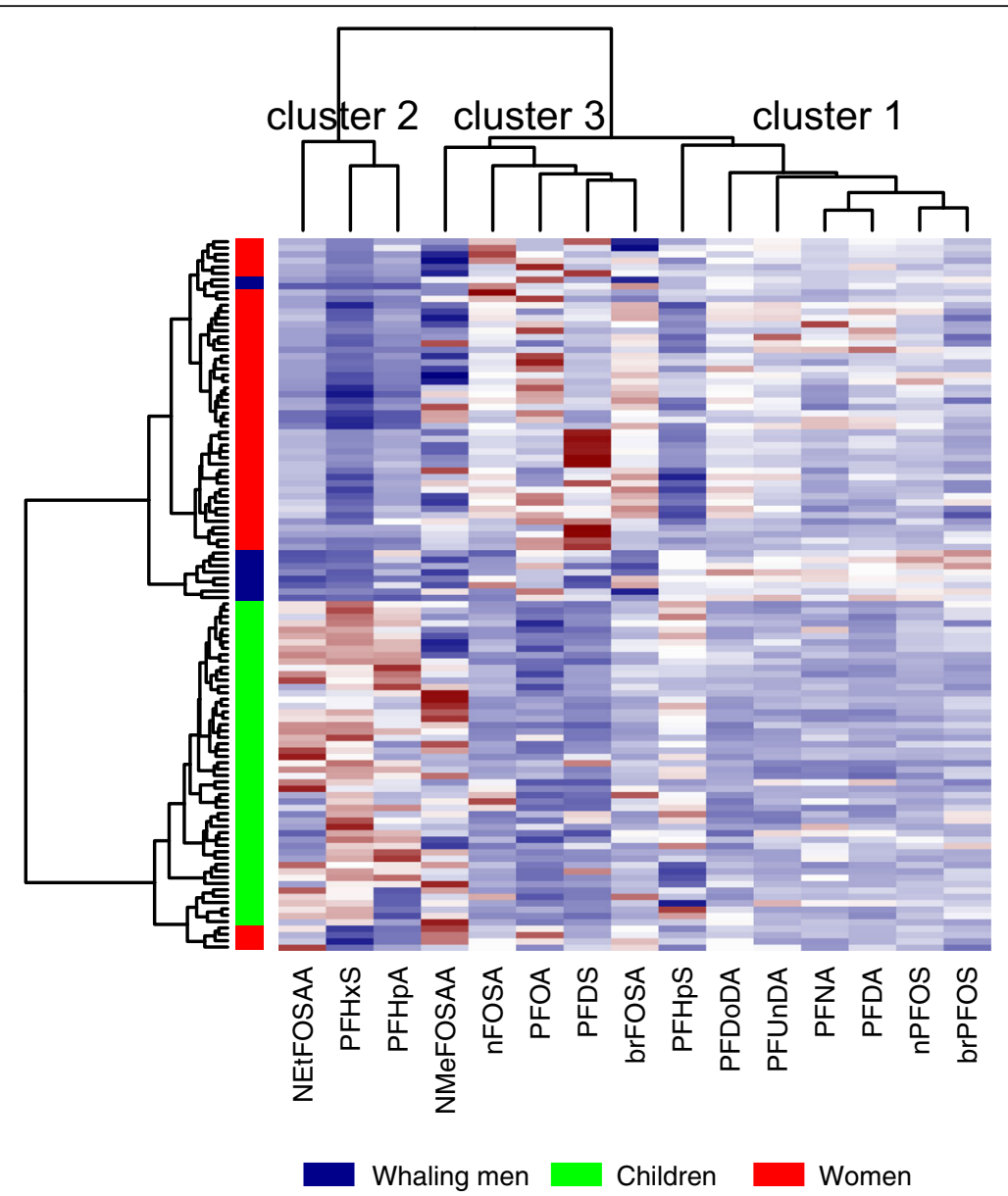

Fig. 3 Hierarchical clustering of serum profiles measured in Faroese whaling men, children and women. Each row represents an individual and each column represents a PFAS. Red (high) and blue (low) shades represent compositions of each PFAS in each individual relative to other individuals

PFDA, after adjusting for socio-demographic factors (Additional file 1: Table S5) and other exposure sources including floor type, drinking water sources, fire-fighting foam exposure (generalized additive model, $p<0.05$, Fig. 5). Fully or partially carpet covered floors were associated with $37.2 \%$ increase in serum PFHxS concentrations, $17.2 \%$ increase in N-MeFOSAA, and $12.2 \%$ increases in PFOS $(p<0.05$, Additional file 1 Table S5). The final multivariable model explains $9-25 \%$ of the variance in PFAS concentrations for the six PFASs considered in the NHANES 2005-06 serum data examined here (Additional file 1 Table S5 and Fig. S5).

Unadjusted associations of potential predictors with serum PFAS concentrations measured in the CHirP study are shown in Additional file 1: Table S4. The final model explains $34-58 \%$ of the variance in PFAS concentrations for the four PFASs considered in CHirP (Additional file 1: Table S6 and Figure S6). Similar to NHANES, we found statistically significantly positive associations between shellfish consumption and increased
PFNA concentrations in CHirP, but not other PFASs. We found positive associations between serum PFHxS composition and increased use of stovetop Teflon cookware and preheated packaged foods (Fig. 6). PFOS was also associated with increased use of packaged foods. These results were adjusted for PFAS exposure sources from other consumer goods, seafood consumption and demographic factors that could affect toxicokinetics such as race/ethnicity, previous pregnancy and breastfeeding history (Additional file 1: Table S6).

\section{Discussion}

Our results link the chemical fingerprint of 15 PFASs in human serum with predominant exposure sources using measurements collected at similar times for three demographic groups in the Faroe Islands. Our analysis suggests that individuals exposed to PFASs from marine food are identifiable by an elevated proportion of C9C12 PFCAs in their serum. Individuals who have exposures dominated by consumer products have relatively 


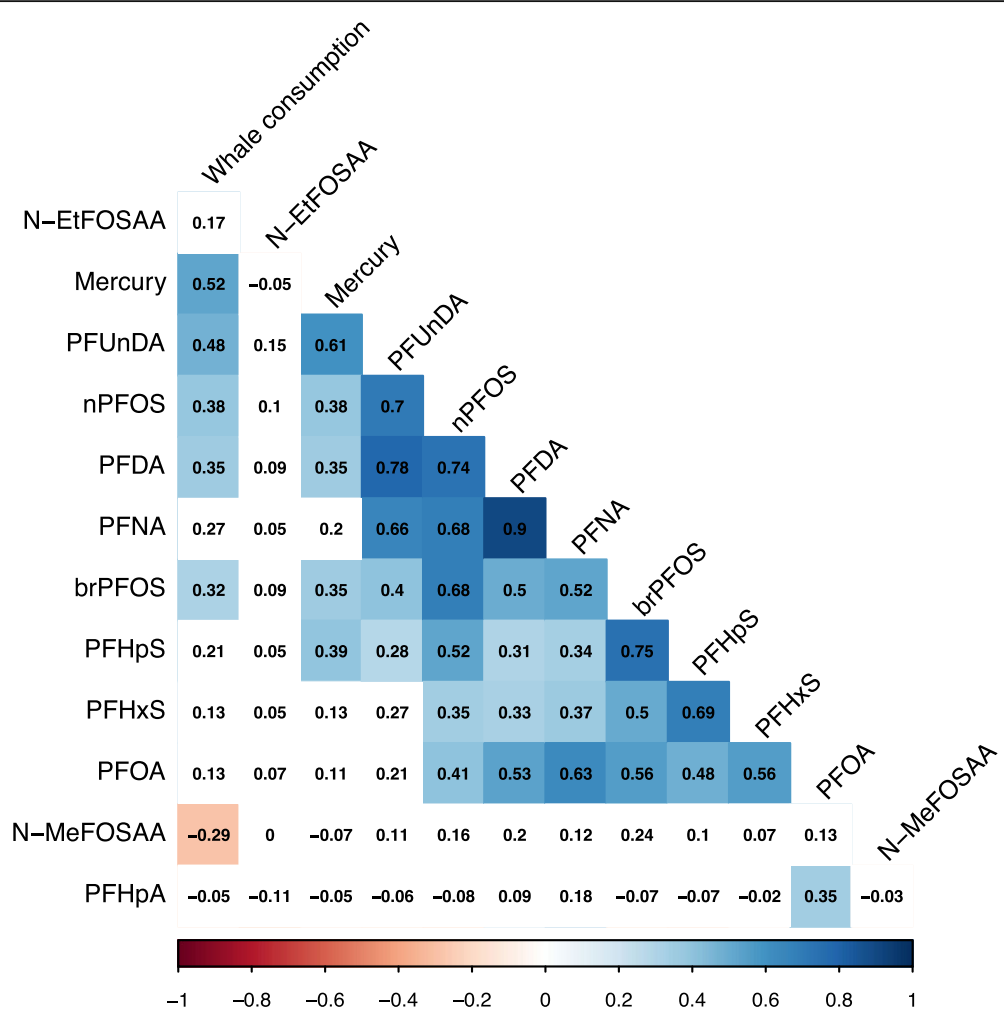

Fig. 4 Spearman correlation matrix between hair-mercury concentrations as an indicator for seafood consumption, dietary recall data on whale consumption, and PFAS concentrations in serum from Faroese children. Statistically significant correlation coefficients are indicated by blue (positive) and red (negative) shades $(p<0.05)$. Color intensities correspond to the strength of the association

higher proportions of PFHxS, and N-EtFOSAA. We find the same chemical fingerprints are associated with the same exposure sources in the NHANES and CHirP cohorts, suggesting generalizability of results from the Faroese population.

C9-C12 PFCAs are known to bioaccumulate in aquatic food webs [41, 43, 58]. Pilot whale meat has similar composition to other seafood $[40,41,43]$. One exception is a relatively higher composition of FOSA in whale meat [42]. However, FOSA is biotransformed in humans, resulting in elevated exposures to PFOS, similar to other seafood [14, 24, 59]. This is consistent with our results (first PCA component) suggesting that C9-C12 PFCAs are effective tracers of seafood consumption. The first PCA component separates whaling men from the other Faroese groups known to consume less whale meat. Some of the compounds in this cluster (PFOS and PFOA) are commonly found in a multitude of exposure sources and thus do not serve as effective tracers. This is reflected by differences with hierarchical clustering results (Fig. 3).

Strong positive correlations between PFHpS, PFDA, PFOS, PFUnDA and hair mercury concentrations among Faroese children reinforce seafood as a common exposure source. The poor correlation between hair mercury and several other PFASs, such as PFHxS, N-MeFOSAA and N-EtFOSAA, suggests that seafood consumption patterns do not drive serum concentrations of these compounds. These results are consistent with findings from PCA and hierarchical clustering results.

In both NHANES 2005-06 and the CHirP cohort, PFNA was associated with increased seafood consumption. PFOS and PFOA are ubiquitously found in multiple exposure sources and are thus not robust tracers for any specific source. PFDA and PFUnDA were not detectable in more than $50 \%$ of study population in CHirP, therefore we did not investigate their association with shellfish consumption. Our results are consistent with Falandysz et al. [59] who first reported elevated PFAS exposures among seafood consumers on the Baltic Coast. Similarly, Haug et al. [13] estimated that seafood accounts for $93 \%$ of daily PFUnDA intake and $81 \%$ of PFOS for adults in Norway. Positive associations between seafood consumption and serum PFDA, PFNA, PFOS and PFUnDA concentrations have also been reported for NHANES data from recent years (2007-2014) [56].

The second PCA component in Fig. 2a consists of PFHxS, PFHpA and two PFOS precursors, N-MeFOSAA and N-EtFOSAA. It separates Faroese children from the other Faroese groups. N-MeFOSAA belongs to a 
PFOS



PFHxS

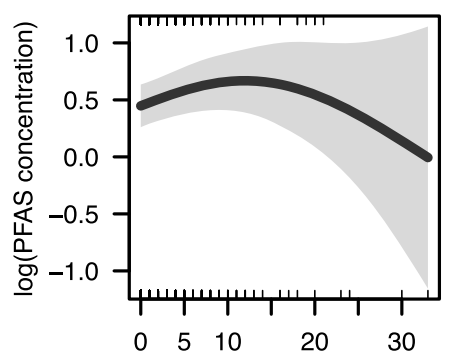

Number of shellfish meals in past $30 \mathrm{~d}$

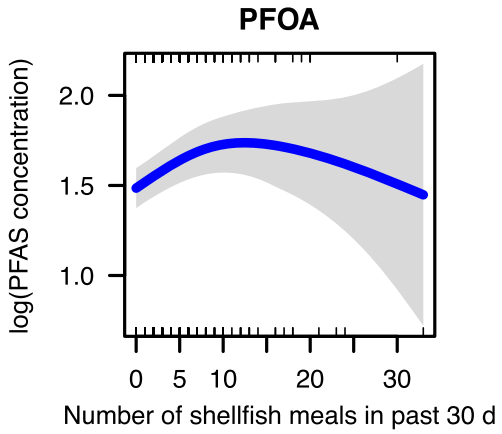

PFNA



PFDA


Fig. 5 Associations between number of shellfish meals consumed in the past 30 days and serum PFAS levels in NHANES $2005-06$ participants. The generalized additive model plots are controlled for age, sex, race/ethnicity, household income, body mass index (BMI), born place, prior pregnancy and breastfeeding, menstrual status, military service, tap water sources, and floor type. The solid line is the estimate and statistical significance is shown by color (blue: $p<0.05$ ). Shaded grey areas represent the $95 \%$ confidence interval

different cluster in hierarchical clustering and is thus less likely to be a robust tracer (Fig. 3). Children have higher PFASs body burdens due to trans-placental transfer, breastfeeding, and frequent hand-to-mouth exposure $[20,26,60]$. Trans-placental transfer and breastfeeding are unlikely to fully account for the high scores on the second component for children in our study. First, the age of children (7 yrs) in this study suggests the influence of trans-placental transfer and breastfeeding on their PFAS body burdens should be small compared to other childhood exposure sources, despite the long halflives of PFHxS (mean 7.3-8.3 years) [61-63]. Second, PFHxS and N-EtFOSAA are not the main compounds known to be transferred in breast milk. Mogensen et al. [20] observed that each month of exclusive breastfeeding was associated with a $30 \%$ increase in serum PFOS,
PFOA, PFNA, and PFDA in Faroese children. In the C8 Science Panel Study, Mondal et al. [64] reported a monotonic decline in maternal serum concentrations as the duration of breastfeeding increased for PFOS, PFOA and PFNA, but not for PFHxS. Therefore, we infer that elevated exposures to PFHxS and other compounds in the second component in Faroese children reflect other postnatal sources.

Several prior surveys have identified PFHxS as one of the most abundant PFASs present in indoor dust samples $[65,66]$. N-MeFOSAA and N-EtFOSAA have been detected in human serum following exposure to the volatile precursors N-methyl perfluorooctane sulfonamidoethanol (N-MeFOSE) and N-ethyl perfluorooctane sulfonamidoethanol (N-EtFOSE) [67, 68]. High levels (>1000 ppm) of N-MeFOSE and PFHxS were 
PFOS

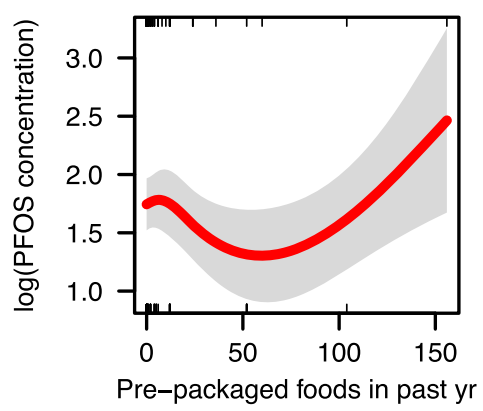

PFNA

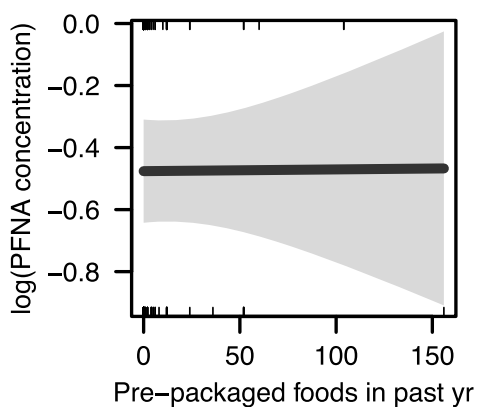

PFOA

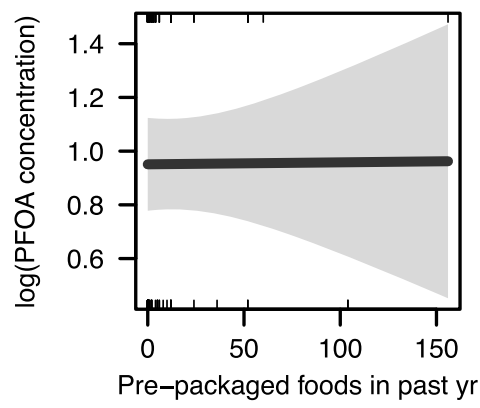

PFHXS

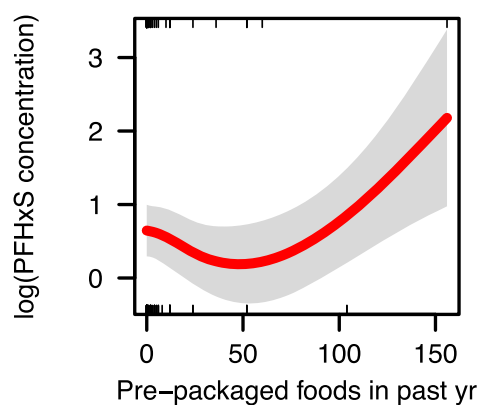

Fig. 6 Associations between number of preheated packaged food consumed in the previous year and serum PFAS levels in CHirP participants. The generalized additive model plots are controlled for age, race/ethnicity, prior pregnancy and breastfeeding, seafood consumption and other consumer product use including carpet cleaner, fire-fighting foam, wax, non-stick cookware, etc. The solid line is the estimate and statistical significance is shown by color (red: $p<0.05$ ). Shaded grey areas represent the $95 \%$ confidence interval

reported in several 3M Scotchguard formulations [69]. N-EtFOSE was mainly used in food packaging during the time period of sample collection in this study and was detected in indoor air samples [18]. PFHxS was systematically underestimated in prior studies that modeled PFAS exposures in children through placental transfer and breastfeeding, suggesting the importance of missing postnatal exposure sources such as household dust [26]. We thus propose that N-EtFOSAA and PFHxS are both tracers of exposures that originate from consumer products.

Recent studies have reported a high prevalence of fluorinated chemicals in food packaging material [70], although potential contributions to serum-PFAS concentrations have not been quantified. Positive associations between N-EtFOSAA and PFHxS and use of carpet, non-stick cookware and food packaging were apparent in analyses using NHANES 2005-06 serum data and the CHirP cohort. Positive associations between serum PFOS concentrations and carpet use were observed in NHANES 2005-06 and between serum PFOS and use of packaged foods in the CHirP study. Migration of N-EtFOSE based chemicals from carpet cleaning products and food packaging materials and subsequent transformation to PFOS may explain these results $[10,11]$.
Household dust from the Faroe Islands has similar PFAS profiles to samples from Canada, Spain, Australia [71]. Our findings are consistent with other environmental monitoring studies that have linked higher PFHxS levels in indoor dust and FOSAs/FOSEs levels in indoor air to carpet area $[66,68,71]$. A recent study of Boston area children found elevated PFOS, PFHxS and NMeFOSAA in sera from individuals who sleep in a room with carpet or a rug [60]. Other studies have found positive associations between PFOS and PFHxS and use of stain-repellant clothing, firefighting foams and consumption of microwavable foods and negative associations between PFHxS and vacuuming frequency [72, 73]. Data linking consumer product use to serum PFAS levels are scarce due to challenges associated with quantifying long-term user behavior.

By comparing Faroese groups with NHANES individuals of the same gender/race and similar age collected around the same time periods, our analysis reduces variability in serum PFAS profiles due temporal and toxicokinetic differences (Fig. 2b-d). Faroese men and children have higher scores on the PCA Component 1, which mainly consist of C9-C12 PFCAs, compared to their NHANES counterpart. This is consistent with frequent seafood consumption in the Faroes and our hypothesis that these PFASs can be tracers for exposure 
from seafood consumption. Statistical clusters for the two populations of 13-year old children (Fig. 2c) overlap more than the whaling men and NHANES men (Fig. 2b), suggesting greater similarity in exposure sources. These results suggest greater relative exposure from consumer products in children from the Faroe Islands compared to whaling men.

Serum samples from Faroese mothers were obtained two weeks after childbirth. Previous studies have found parous women have lower PFASs compared to nulliparous women, and PFASs levels increase with the time interval since the last pregnancy and breastfeeding period $[74,75]$. We compared Faroese mothers to NHANES women who were within one year of postpartum because finer resolution data on time since delivery is not available in NHANES. The salient differences in PFASs profiles may thus reflect differences in the postpartum period for Faroese mothers (two weeks) compared to NHANES women.

\section{Conclusions}

Recent advances in analytical chemistry have allowed numerous PFASs to be reliably detected in small volumes of human serum [39]. The suite of 15 PFASs reported in this study goes beyond standard epidemiological studies that typically report five or fewer compounds. Including additional PFASs and metabolites of precursors would increase the probability of identifying unique biomarkers for human exposure pathways [76-78]. Statistical methods presented in this paper are useful for qualitative differentiation of dominant exposure sources. This method could be enhanced by using factor analysis and receptor models to quantify the relative contribution of different sources but requires a metric for total PFAS exposure [79]. Routine measurements of extractable organic fluorine in human sera might thus complement data on individual PFASs and enrich the exposure information derived from serum samples [80-82].

Recent epidemiological research on associations between PFAS exposures and adverse human health effects has emphasized correlations with single compounds. Due to inter-correlations between PFASs, adjusted regression analyses are often non-informative. One approach for accounting for complex exposures is to apply structural equation modeling. In this approach, individual serum-PFAS concentrations contribute to a latent PFAS variable, with different loading factors. Our results show that this approach is useful but can be improved by a weighting of PFASs that reflects exposure sources. PFASs serum profiles will inform a better understanding of the health impact of PFASs with different origins. The present study illustrates that this approach is feasible and that addition of a few more PFASs than those routinely measured will make this possible.
Our analyses suggest that the composition of PFASs in human sera contains important information on dominant PFASs exposure sources that can be leveraged from samples that may be routinely collected during epidemiological studies. Individuals exposed to PFASs from seafood are identifiable by an elevated proportion of C9. C12 PFCAs in their serum, while those exposed to consumer products have relatively higher proportions of PFHxS and N-EtFOSAA. Some PFASs such as PFOS and PFOA are more ubiquitous in many exposure sources and thus do not serve as effective tracers. Routine reporting of more than the five or six PFASs that are now standard for epidemiological studies would be useful for making such inferences. Confounding factors such as temporal trends and toxicokinetic processes must be carefully considered before making inferences about differences in exposure sources.

\section{Additional file}

Additional file 1: Supplementary figures, tables and information. (PDF $1110 \mathrm{~kb}$ )

\section{Abbreviations}

ANOVA: Analysis of variance; CDC: Center for Disease Control and Prevention CHirP: Chemicals, Health and Pregnancy; FOSA: Perfluorooctane sulfonamide; GAM: Generalized additive model; HPLC-MS/MS: High-pressure liquid chromatography with tandem mass spectrometry; HSD: Honestly significant difference; LOD: Limit of detection; N-EtFOSAA: N-ethyl perfluorooctane sulfonamidoacetate; N-EtFOSE: N-ethyl perfluorooctane sulfonamidoethanol; NHANES: National Health and Nutrition Examination Survey; N-MeFOSAA: Nmethyl perfluorooctane sulfonamidoacetate; N-MeFOSE: N-methyl perfluorooctane sulfonamidoethanol; PCA: Principal components analysis; PFAA: Perfluoroalkyl acid; PFASs: Poly- and perfluoroalkyl substances; PFBA: Perfluorobutanoic acid; PFBS: Perfluorobutanesulfonic acid; PFCAs: Perfluoroalkyl carboxylates; PFDA: Perfluorodecanoic acid; PFDoDA: Perfluorododecanoic acid; PFDS: Perfluorodecanesulfonic acid; PFHpA: Perfluoroheptanoic acid; PFHpS: Perfluoroheptanesulfonic acid; PFHXA: Perfluorohexanoic acid; PFHxS: Perfluorohexanesulfonic acid; PFNA: Perfluorononanoic acid; PFOA: Perfluorooctanoic acid;

PFOS: Perfluorooctanesulfonic acid; PFPeA: Perfluoropentanoic acid; PFUnDA: Perfluoroundecanoic acid; ROS: Robust regression on order statistics

\section{Acknowledgements}

We thank Dr. Brent Coull from Harvard University for assistance with statistical methods.

\section{Funding}

This work was supported by the Richard and Susan Smith Foundation, the U.S. National Science Foundation Office of Polar Programs (PLR 1203496), the NSF-NIH Oceans and Human Health Program (OCE-1321612) and National Institute of Environmental Health Sciences grants ES012199, ES013692 and ES021993. XCH, PG and EMS are also supported by P42ES027706.

\section{Availability of data and materials}

We were granted access to the Faroese data and the Canadian pregnancy cohort (CHirP) for this specific analysis. We are not authorized to share the human data with other investigators. Data on NHANES can be downloaded from Center for Disease Control and Prevention website https://

wwwn.cdc.gov/nchs/nhanes/Default.aspx

\section{Authors' contributions}

$\mathrm{XCH}, \mathrm{PG}$ and EMS designed the study. XCH was responsible for data analyses, visualization, and interpretation of the results. PG and PW collected 
data and blood samples from the Faroese cohorts. FN performed serum PFAS measurements. GMW collected data for the CHirP cohort. CD, XZ, PG and EMS contributed to the data analysis and interpretation of results. $\mathrm{XCH}$ and EMS wrote the manuscript. All authors provided editorial feedback and approved the final manuscript.

\section{Ethics approval and consent to participate}

All participants provided written consent, and study protocols were reviewed and approved by the Faroese ethical review committee and the institutional review board in the U.S.

\section{Consent for publication}

Not applicable

\section{Competing interests}

The authors declare that they have no competing interests.

\section{Publisher's Note}

Springer Nature remains neutral with regard to jurisdictional claims in published maps and institutional affiliations.

\section{Author details}

${ }^{1}$ Harvard T.H. Chan School of Public Health, Boston, MA 02215, USA. ${ }^{2}$ Harvard John A. Paulson School of Engineering and Applied Sciences, Harvard University, 128 Pierce Hall, Cambridge, MA 02138, USA. ${ }^{3}$ University of Southern Denmark, DK-5000 Odense, Denmark. ${ }^{4}$ The Faroese Hospita System, FR-100 Tórshavn, Faroe Islands. ${ }^{5}$ Simon Fraser University, Burnaby, BC V5A 1S6, Canada.

Received: 17 October 2017 Accepted: 23 January 2018 Published online: 01 February 2018

\section{References}

1. Kato K, Wong L-Y, Jia LT, Kuklenyik Z, Calafat AM. Trends in exposure to Polyfluoroalkyl Chemicals in the U.S. population: 1999-2008. Environ. Sci. Technol. 2011;45(19):8037-45.

2. Nøst TH, Vestergren R, Berg V, Nieboer E, Odland J $\varnothing$, Sandanger TM. Repeated measurements of per- and polyfluoroalkyl substances (PFASs) from 1979 to 2007 in males from northern Norway: assessing time trends, compound correlations and relations to age/birth cohort. Environ Int. 2014; 67:43-53.

3. Haines DA, Khoury C, Saravanabhavan G, Werry K, Walker M, Malowany M. Human biomonitoring reference values derived for persistent organic pollutants in blood plasma from the Canadian health measures survey 2007-2011. Int J Hyg Environ Health. 2017;220(4):744-56.

4. Zhou Z, Shi Y, Vestergren R, Wang T, Liang Y, Cai Y. Highly elevated serum concentrations of Perfluoroalkyl substances in fishery employees from Tangxun Lake, China. Environ. Sci. Technol. 2014;48(7):3864-74.

5. Toms L-M, Thompson J, Rotander A, Hobson P, Calafat AM, Kato K, Ye X, Broomhall S, Harden F, Mueller JF. Decline in perfluorooctane sulfonate and perfluorooctanoate serum concentrations in an Australian population from 2002 to 2011. Environ Int. 2014;71:74-80.

6. Harada $\mathrm{KH}$, Yang H-R, Moon C-S, Hung NN, Hitomi T, Inoue K, Niisoe T, Watanabe T, Kamiyama S, Takenaka K. Levels of perfluorooctane sulfonate and perfluorooctanoic acid in female serum samples from Japan in 2008, Korea in 1994-2008 and Vietnam in 2007-2008. Chemosphere. 2010;79(3): 314-9.

7. Grandjean P, Budtz-Jorgensen E. Immunotoxicity of perfluorinated alkylates: calculation of benchmark doses based on serum concentrations in children. Environ Health. 2013;12(1):35.

8. Vaughn B, Winquist A, Steenland K. Perfluorooctanoic acid (PFOA) exposures and incident cancers among adults living near a chemical plant. Environ Health Perspect. 2013;121(11-12):1313.

9. Grandjean P. Clapp R. Perfluorinated alkyl substances. New Solut. 2015;25(2): 147-63

10. Martin JW, Asher BJ, Beesoon S, Benskin JP, Ross MS. PFOS or PreFOS? Are perfluorooctane sulfonate precursors (PreFOS) important determinants of human and environmental perfluorooctane sulfonate (PFOS) exposure? J Environ Monit. 2010;12(11):1979-2004.
11. D'eon JC, Mabury SA. Is indirect exposure a significant contributor to the burden of Perfluorinated acids observed in humans? Environ Sci Technol. 2011;45(19):7974-84

12. Scheringer $M$, Trier $X$, Cousins IT, de Voogt $P$, Fletcher $T$, Wang $Z$, Webster TF. Helsingør statement on poly- and perfluorinated alkyl substances (PFASs). Chemosphere 2014, 114(0):337-339.

13. Haug LS, Thomsen C, Brantsæter AL, Kvalem HE, Haugen M, Becher G, Alexander J, Meltzer HM, Knutsen HK. Diet and particularly seafood are major sources of perfluorinated compounds in humans. Environ Int. 2010; 36(7):772-8.

14. Weihe P, Kato K, Calafat AM, Nielsen F, Wanigatunga AA, Needham LL, Grandjean P. Serum concentrations of Polyfluoroalkyl compounds in Faroese whale meat consumers. Environ. Sci. Technol. 2008;42(16):6291-5.

15. Denys S, Fraize-Frontier S, Moussa O, Bizec BL, Veyrand B, Volatier J-L. Is the fresh water fish consumption a significant determinant of the internal exposure to perfluoroalkylated substances (PFAS)? Toxicol Lett. 2014;231(2): 233-8.

16. Zhang T, Sun HW, Wu Q, Zhang XZ, Yun SH, Kannan K. Perfluorochemicals in meat, eggs and indoor dust in China: assessment of sources and pathways of human exposure to perfluorochemicals. Environ. Sci. Technol. 2010:44(9):3572-9

17. Jogsten IE, Perelló G, Llebaria X, Bigas E, Martí-Cid R, Kärrman A, Domingo J. Exposure to perfluorinated compounds in Catalonia, Spain, through consumption of various raw and cooked foodstuffs, including packaged food. Food Chem Toxicol. 2009;47(7):1577-83.

18. Shoeib M, Harner T, Wilford BH, Jones KC, Zhu J. Perfluorinated sulfonamides in indoor and outdoor air and indoor dust: occurrence, partitioning, and human exposure. Environ Sci Technol. 2005:39(17):6599-606.

19. Zhang T, Sun H, Lin Y, Qin X, Zhang Y, Geng X, Kannan K. Distribution of poly-and perfluoroalkyl substances in matched samples from pregnant women and carbon chain length related maternal transfer. Environ. Sci. Technol. 2013:47(14):7974-81.

20. Mogensen UB, Grandjean P, Nielsen F, Weihe P, Budtz-Jørgensen E. Breastfeeding as an exposure pathway for perfluorinated alkylates. Environ. Sci. Technol. 2015;49(17):10466-73.

21. Landsteiner A, Huset C, Williams A, Johnson J. Biomonitoring for Perfluorochemicals in a Minnesota community with known drinking water contamination. J Environ Health. 2014;77(5):14-9.

22. Hoffman K, Webster TF, Bartell SM, Weisskopf MG, Fletcher T, Vieira VM. Private drinking water wells as a source of exposure to perfluorooctanoic acid (PFOA) in communities surrounding a fluoropolymer production facility. Environ. Health Perspect. 2010;119(1):92-97.

23. Lorber M, Egeghy PP. Simple intake and pharmacokinetic modeling to characterize exposure of Americans to perfluoroctanoic acid, PFOA. Environ. Sci. Technol. 2011:45(19):8006-14.

24. Tittlemier SA, Pepper K, Seymour C, Moisey J, Bronson R, Cao X-L, Dabeka RW. Dietary exposure of Canadians to perfluorinated carboxylates and perfluorooctane sulfonate via consumption of meat, fish, fast foods, and food items prepared in their packaging. J Agric Food Chem. 2007;55(8): 3203-10.

25. Worley RR, Yang X, Fisher J. Physiologically based pharmacokinetic modeling of human exposure to perfluorooctanoic acid suggests historical non drinking-water exposures are important for predicting current serum concentrations. Toxicol Appl Pharmacol. 2017;330(Supplement C):9-21.

26. Verner M-A, Ngueta G, Jensen ET, Fromme H, Völkel W, Nygaard UC, Granum B, Longnecker MP. A simple pharmacokinetic model of prenata and postnatal exposure to Perfluoroalkyl substances (PFASs). Environ. Sci. Technol. 2016:50(2):978-86.

27. Wang Z, Cousins IT, Scheringer M, Hungerbühler K. Fluorinated alternatives to long-chain perfluoroalkyl carboxylic acids (PFCAs), perfluoroalkane sulfonic acids (PFSAs) and their potential precursors. Environ Int. 2013;60: 242-8.

28. Johnson GW, Ehrlich R, Full W, Ramos S. Principal components analysis and receptor models in environmental forensics. San Diego: Academic Press; 2002.

29. Wang Z, Stout SA, Fingas M. Forensic fingerprinting of biomarkers for oil spill characterization and source identification. Environ Forensic. 2006;7(2):105-46.

30. Zhang X, Diamond ML, Robson M, Harrad S. Sources, emissions, and fate of polybrominated diphenyl ethers and polychlorinated biphenyls indoors in Toronto, Canada. Environ. Sci. Technol. 2011;45(8):3268-74.

31. Zhang X, Lohmann R, Dassuncao C, Hu XC, Weber AK, Vecitis CD, Sunderland EM. Source attribution of poly- and Perfluoroalkyl substances 
(PFASs) in surface waters from Rhode Island and the New York metropolitan area. Environ Sci Tech Let. 2016;3(9):316-21.

32. Grandjean P, Andersen E, Budtz-Jørgensen E, et al. Serum vaccine antibody concentrations in children exposed to perfluorinated compounds. JAMA. 2012;307(4):391-7.

33. Webster GM: Exposure sources and thyroid effects of perfluorinated compounds (PFCs) during pregnancy: results from the chemicals, health and pregnancy study (CHirP). Vancouver, Canada: The University Of British Columbia; 2011.

34. National Health and Nutrition Examination Survey 2005-2006 [https:// wwwn.cdc.gov/nchs/nhanes/Search/Nhanes05_06.aspx].

35. Kim B-M, Choi AL, Ha E-H, Pedersen L, Nielsen F, Weihe P, Hong Y-C, BudtzJørgensen $E$, Grandjean P. Effect of hemoglobin adjustment on the precision of mercury concentrations in maternal and cord blood. Environ Res. 2014;132:407-12.

36. Timmermann CAG, Budtz-Jørgensen E, Petersen MS, Weihe P, Steuerwald U, Nielsen F, Jensen TK, Grandjean P. Shorter duration of breastfeeding at elevated exposures to perfluoroalkyl substances. Reprod Toxicol. 2016;68: 164-70.

37. Li M, von Stackelberg K, Rheinberger CM, Hammitt JK, Krabbenhoft DP, Yin $R$, Sunderland EM. Insights from mercury stable isotopes into factors affecting the internal body burden of methylmercury in frequent fish consumers. Elem Sci Anth. 2016;4:103.

38. Debes F, Budtz-Jorgensen E, Weihe P, White RF, Grandjean P. Impact of prenatal methylmercury exposure on neurobehavioral function at age 14 years. Neurotoxicol Teratol. 2006;28(5):536-47.

39. Haug LS, Thomsen C, Becher G. A sensitive method for determination of a broad range of perfluorinated compounds in serum suitable for large-scale human biomonitoring. J Chromatogr A. 2009;1216(3):385-93.

40. Tomy GT, Budakowski W, Halldorson T, Helm PA, Stern GA, Friesen K, Pepper K, Tittlemier SA, Fisk AT. Fluorinated organic compounds in an eastern Arctic marine food web. Environ. Sci. Technol. 2004;38(24):6475-81.

41. Tomy GT, Pleskach K, Ferguson SH, Hare J, Stern G, Maclnnis G, Marvin CH, Loseto L. Trophodynamics of some PFCs and BFRs in a western Canadian Arctic marine food web. Environ. Sci. Technol. 2009;43(11):4076-81.

42. Dassuncao C, Hu XC, Zhang X, Bossi R, Dam M, Mikkelsen B, Sunderland EM. Temporal shifts in poly- and Perfluoroalkyl substances (PFASs) in North Atlantic pilot whales indicate large contribution of atmospheric precursors. Environ. Sci. Technol. 2017;51(8):4512-21.

43. Kelly BC, Ikonomou MG, Blair JD, Surridge B, Hoover D, Grace R, Gobas FAPC. Perfluoroalkyl contaminants in an Arctic marine food web: Trophic magnification and wildlife exposure. Environ. Sci. Technol. 2009;43(11):4037-43.

44. Yoo H, Yamashita N, Taniyasu S, Lee KT, Jones PD, Newsted JL, Khim JS, Giesy JP. Perfluoroalkyl acids in marine organisms from Lake Shihwa, Korea. Arch Environ Contam Toxicol. 2009:57(3):552-60.

45. National Health and Nutrition Examination Survey 2005-2006 Data Documentation, Codebook, and Frequencies [https://wwwn.cdc.gov/Nchs/ Nhanes/2005-2006/PFC_D.htm - Description_of_Laboratory_Methodology]

46. Webster GM, Teschke K, Janssen PA. Recruitment of healthy first-trimester pregnant women: lessons from the chemicals, Health \& Pregnancy study (CHirP). Matern Child Health J. 2012;16(2):430-8.

47. Webster GM, Venners SA, Mattman A, Martin JW. Associations between perfluoroalkyl acids (PFASs) and maternal thyroid hormones in early pregnancy: a population-based cohort study. Environ Res. 2014;133:338-47.

48. Kuklenyik Z, Reich JA, Tully JS, Needham LL, Calafat AM. Automated solidphase extraction and measurement of Perfluorinated organic acids and amides in human serum and milk. Environ. Sci. Technol. 2004;38(13):3698704.

49. Lee L, Helsel D. Statistical analysis of water-quality data containing multiple detection limits: S-language software for regression on order statistics. Comput Geosci. 2005;31(10):1241-8.

50. Lopaka Lee: NADA: Nondetects And Data Analysis for environmental data. In: R package version 15-6. 2013.

51. Mendiburu Fd: agricolae: Statistical Procedures for Agricultural Research. In: R package version 12-4. 2016.

52. Lê S, Josse J, Husson F. FactoMineR: an R package for multivariate analysis. J Stat Softw. 2008;25(1):1-18.

53. R Core Team: R: A Language and Environment for Statistical Computing. In. Edited by R Foundation for Statistical Computing. Vienna, Austria; 2016.

54. Clifton Dassuncao RB, Maria Dam, Philippe Grandjean, Xindi C. Hu, Bjarni Mikkelsen, Xianming Zhang, Elsie M. Sunderland: Poly and perfluoroalkyl substances (PFASs) in a marine food web and implications for human exposure. In: Society of Environmental Toxicology and Chemistry North American Annual Meeting. Orlando, FL.; 2016.

55. Nøst TH, Vestergren $R$, Berg $V$, Nieboer $E$, Odland J $\varnothing$, Sandanger TM. Repeated measurements of per- and polyfluoroalkyl substances (PFASs) from 1979 to 2007 in males from northern Norway: assessing time trends, compound correlations and relations to age/birth cohort. Environ Int. 2014; 67(Supplement C):43-53.

56. Christensen KY, Raymond M, Blackowicz M, Liu Y, Thompson BA, Anderson HA, Turyk M. Perfluoroalkyl substances and fish consumption. Environ Res. 2017;154:145-51.

57. Hastie T: gam: Generalized Additive Models. R package version 1.14. In.; 2016

58. Ng CA, Hungerbühler K. Bioaccumulation of perfluorinated alkyl acids: observations and models. Environ. Sci. Technol. 2014;48(9):4637-48.

59. Falandysz J, Taniyasu S, Gulkowska A, Yamashita N, Schulte-Oehlmann U. Is fish a major source of fluorinated surfactants and repellents in humans living on the Baltic coast? Environ. Sci. Technol. 2006;40(3):748-51.

60. Harris MH, Rifas-Shiman SL, Calafat AM, Ye X, Mora AM, Webster TF, Oken E, Sagiv SK. Predictors of Per- and Polyfluoroalkyl Substance (PFAS) Plasma Concentrations in 6-10 Year Old American Children. Environ Sci Technol. 2017;51(9):5193-204.

61. Spliethoff HM, Tao L, Shaver SM, Aldous KM, Pass KA, Kannan K, Eadon GA. Use of newborn screening program blood spots for exposure assessment: declining levels of Perfluorinated compounds in New York state infants. Environ. Sci. Technol. 2008;42(14):5361-7.

62. Olsen GW, Burris JM, Ehresman DJ, Froehlich JW, Seacat AM, Butenhoff JL, Zobel LR. Half-life of serum elimination of Perfluorooctanesulfonate, Perfluorohexanesulfonate, and Perfluorooctanoate in retired Fluorochemical production workers. Environ Health Perspect. 2007;115(9):1298-305.

63. Zhang Y, Beesoon S, Zhu L, Martin JW. Biomonitoring of Perfluoroalkyl acids in human urine and estimates of biological half-life. Environ. Sci. Technol. 2013;47(18):10619-27.

64. Mondal D, Weldon RH, Armstrong BG, Gibson LJ, Lopez-Espinosa M-J, Shin H-M, Fletcher T. Breastfeeding: a potential excretion route for mothers and implications for infant exposure to perfluoroalkyl acids. Environ Health Perspect. 2014;122(2):187.

65. Strynar MJ, Lindstrom AB. Perfluorinated compounds in house dust from Ohio and North Carolina, USA. Environ. Sci. Technol. 2008;42(10): 3751-6.

66. Karásková P, Venier M, Melymuk L, Bečanová J, Vojta Š, Prokeš R, Diamond ML, Klánová J. Perfluorinated alkyl substances (PFASs) in household dust in Central Europe and North America. Environ Int. 2016;94:315-24.

67. Robinson S: Commercial Fluorosurfactants and Their Metabolites in Human Blood. 2011.

68. Fraser AJ, Webster TF, Watkins DJ, Nelson JW, Stapleton HM, Calafat AM, Kato K, Shoeib M, Vieira VM, McClean MD. Polyfluorinated compounds in serum linked to indoor air in office environments. Environ. Sci. Technol. 2012:46(2):1209-15.

69. 3M Company: Fraunhofer ITEM. 28 Day Repeated Dermal Contact Study of $3 \mathrm{M}$ Test Articles in Sprague-Dawley. Appendix A-1. Determination of fluorochemicals residuals in products and products treated webs. In: Final Report 01 G 00002. 69M. Company; 2000.

70. Schaider LA, Balan SA, Blum A, Andrews DQ, Strynar MJ, Dickinson ME, Lunderberg DM, Lang JR, Peaslee GF. Fluorinated Compounds in U.S. Fast Food Packaging. Environmental Science \& Technology Letters. 2017;4(3): 105-11.

71. Eriksson U, Kärrman A. World-wide indoor exposure to polyfluoroalkyl phosphate esters (PAPs) and other PFASs in household dust. Environmental Science \& Technology. 2015;49(24):14503-511.

72. Wu X, Bennett DH, Calafat AM, Kato K, Strynar M, Andersen E, Moran RE, Tancredi DJ, Tulve NS, Hertz-Picciotto I. Serum concentrations of perfluorinated compounds (PFC) among selected populations of children and adults in California. Environ Res. 2015;136:264-73.

73. Siebenaler R, Cameron R, Butt CM, Hoffman K, Higgins CP, Stapleton HM. Serum perfluoroalkyl acids (PFAAs) and associations with behavioral attributes. Chemosphere. 2017;184:687-93.

74. Brantsaeter AL, Whitworth KW, Ydersbond TA, Haug LS, Haugen M, Knutsen HK, Thomsen C, Meltzer HM, Becher G, Sabaredzovic A, et al. Determinants of plasma concentrations of perfluoroalkyl substances in pregnant Norwegian women. Environ Int. 2013;54:74-84. 
75. Wong F, MacLeod M, Mueller JF, Cousins IT. Enhanced elimination of perfluorooctane sulfonic acid by menstruating women: evidence from population-based pharmacokinetic modeling. Environ. Sci. Technol. 2014; 48(15):8807-14.

76. Strynar M, Dagnino S, McMahen R, Liang S, Lindstrom A, Andersen E, McMillan L, Thurman M, Ferrer I, Ball C. Identification of novel Perfluoroalkyl ether carboxylic acids (PFECAs) and Sulfonic acids (PFESAs) in natural waters using accurate mass time-of-flight mass spectrometry (TOFMS). Environ Sci Technol. 2015;49(19):11622-30.

77. Dagnino S, Strynar MJ, McMahen RL, Lau CS, Ball C, Garantziotis S, Webster TF, McClean MD, Lindstrom AB. Identification of biomarkers of exposure to FTOHs and PAPs in humans using a targeted and nontargeted analysis approach. Environ Sci Technol. 2016;50(18):10216-25.

78. Wang Z, DeWitt JC, Higgins CP, Cousins IT. A never-ending story of per- and Polyfluoroalkyl substances (PFASs)? Environ. Sci. Technol. 2017:51(5):2508-18.

79. Thurston GD, Spengler JD. A quantitative assessment of source contributions to inhalable particulate matter pollution in metropolitan Boston. Atmos Environ. 1985;19(1):9-25.

80. Yeung LW, Miyake Y, Taniyasu S, Wang Y, Yu H, So MK, Jiang G, Wu Y, Li J, Giesy JP. Perfluorinated compounds and total and extractable organic fluorine in human blood samples from China. Environ. Sci. Technol. 2008; 42(21):8140-5

81. Miyake Y, Yamashita N, So MK, Rostkowski P, Taniyasu S, Lam PK, Kannan K. Trace analysis of total fluorine in human blood using combustion ion chromatography for fluorine: a mass balance approach for the determination of known and unknown organofluorine compounds. J Chromatogr A. 2007;1154(1):214-21.

82. Yeung LW, Mabury SA. Are humans exposed to increasing amounts of unidentified organofluorine? Environ Chem. 2016;13(1):102-10.

\section{Submit your next manuscript to BioMed Central and we will help you at every step:}

- We accept pre-submission inquiries

- Our selector tool helps you to find the most relevant journal

- We provide round the clock customer support

- Convenient online submission

- Thorough peer review

- Inclusion in PubMed and all major indexing services

- Maximum visibility for your research

Submit your manuscript at www.biomedcentral.com/submit

) Biomed Central 